\section{References}

1. Coselli JS, LeMaire SA. Surgical techniques: thoracoabdominal aorta. Cardiol Clin. 1999;17:751-65.

2. Safi HJ, Miller CC III, Yawn DH, Iliopoulos DC, Subramaniam MH,
Harlin S, et al. Impact of distal aortic and visceral perfusion on liver function during thoracoabdominal and descending thoracic aortic repair. J Vasc Surg. 1998;27(1):145-52.

3. Griepp RB, Ergin MA. Aneurysms of the aortic arch. In: Edmunds LH, editor. Cardiac surgery in the adult. St Louis: Mosby; 1996. p. 1197-224.

\title{
Left atrial dissection after aortic valve replacement
}

\author{
Hiroshi Osawa, MD, PhD, Shinpei Yoshii, MD, PhD, Shigeru Hosaka, MD, PhD, \\ Shoji Suzuki, MD, PhD, Samuel J. K. Abraham, MD, PhD, and Yusuke Tada, MD, PhD, \\ Yamanashi, Japan
}

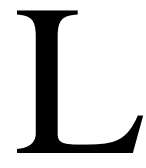

eft atrial dissection is a severe but rare complication occurring in $0.84 \%$ of mitral replacement cases. ${ }^{1}$

We report a case of left atrial dissection caused by aortic annular disruption after aortic valve replacement that was identified by means of transesophageal echocardiography and repaired immediately. Such a case has not been reported in the literature to our knowledge.

\section{Clinical Summary}

A 73-year-old woman underwent aortic valve replacement for aortic stenosis and concomitant coronary artery bypass grafting. Cardiopulmonary bypass was performed with moderate hypothermia and cardioplegic arrest. The left internal thoracic artery was anastomosed to the left anterior descending artery, and a saphenous vein graft was anastomosed to a branch of the obtuse marginal artery. After distal coronary bypass, an aortotomy was made. The aortic valve was found to be calcified, and the calcification extended to the aortic annulus. While removing the calcification, a dissection of the noncoronary annulus occurred and was repaired with everted mattress sutures, with which the prosthetic valve was also fixed. Then a Carbomedics 19-mm prosthetic valve (Sulzer Carbomedics, Austin, Tex) was implanted, and the aortotomy was closed. A proximal saphenous vein graft was anastomosed to the ascending aorta.

After successful weaning from cardiopulmonary bypass, a finding resembling dissection of the left atrium was found on routine intraoperative transesophageal echocardiography (Figure 1). Doppler echocardiography showed a flow originating from the aortic annulus and continuing into the atrial septal wall. The

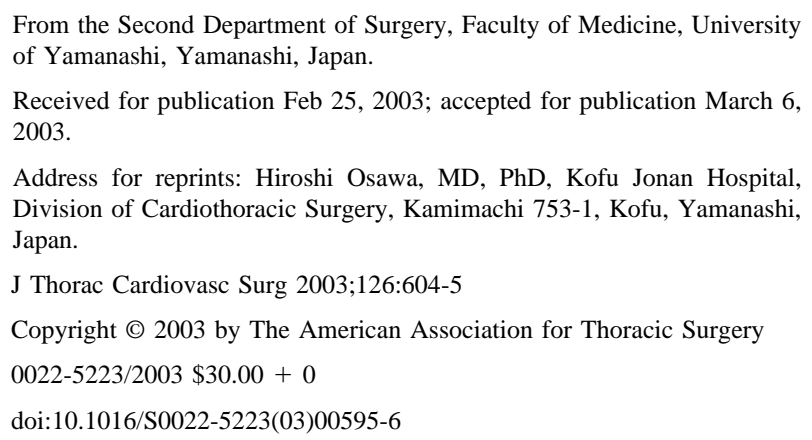

dissection cavity ruptured, and the blood was let out through a defect on the posterior side of the pericardium close to the inferior vena cava. However, the dissection cavity continued to fill up repetitively, and

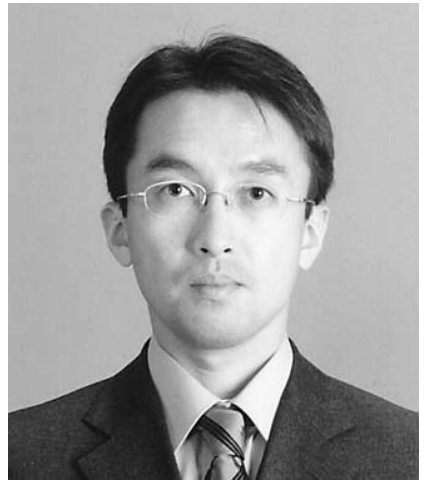

Dr Osawa hemodynamics worsened. We thought the entry might have been made by aortic annular disruption and resulted in dissection of the left atrium, as shown in Figure 2, A.

Cardiopulmonary bypass was reestablished, and an aortotomy was made again. When the prosthetic valve was removed, the noncoronary aortic annulus was divided, and entry into the left atrial dissection was made. The annulus, including the aortic wall, was reconstructed with a Hemashield Gold (Boston Scientific, Natick, Mass) Dacron patch, and aortic valve re-replacement was performed on the reconstructed annulus (Figure 2, B). The dissection cavity was decompressed, and cardiopulmonary bypass was successfully terminated. Postoperative echocardiography demonstrated no abnormal findings of the left atrial wall. The patient was discharged uneventfully, and she is doing well 3 years after the operation.

\section{Discussion}

Left atrial dissection is a rare complication and has been reported to occur in mitral valve surgery to an extent of $0.84 \%$. $^{1}$

In our case aortic annular disruption caused by the removal of annular calcification caused dissection of the left atrium. We examined a cadaver heart and found that division of the noncoronary aortic annulus allowed easy opening into the atrial septal wall, and the wall was comprised of 2 layers running in different directions. The above finding provides convincing evidence that an annular disruption in that site could easily pave the way for a dissection into the wall of the left atrium.

Left atrial dissection is a severe complication that results in low output syndrome and hemodynamic collapse. ${ }^{1,2}$ Most of the previously reported cases have required surgical treatment. ${ }^{1-4}$ It is necessary to close the entry of the dissection to treat this complication. In our case we had to reconstruct the destroyed annulus because the destroyed annulus could not withstand the replacement of the prosthetic valve again. We repaired the annulus with a 


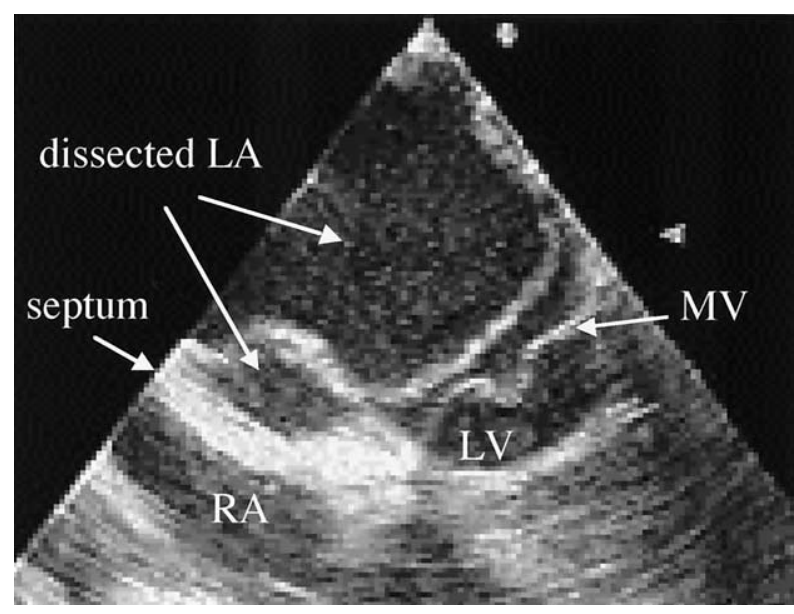

Figure 1. Intraoperative transesophageal echocardiography showing left atrial dissection. $L A$, Left atrium; $L V$, left ventricle; $M V$, mitral valve; $R A$, right atrium.

Dacron patch in the same manner as in the aortic annular extension procedure. In this case retrospectively it is thought that the aortic annulus was relatively small for a $19-\mathrm{mm}$ Carbomedics prosthetic valve. The possibility of the everted mattress suture causing excessive tension to the annulus, consequently resulting in a disruption, also cannot be ruled out. In this case enlarging the aortic annulus before valve replacement or using a supra-annular prosthetic valve, thereby avoiding an everted mattress suture, might have been more suitable.

In our case intraoperative transesophageal echocardiography has proved to be very important. It helped with an immediate diagnosis and prompt correction of the condition. ${ }^{1,2,4,5}$ Transesophageal echocardiography should be used not only in mitral valve surgery but also in aortic surgery.

In conclusion, left atrial dissection could occur after not only mitral valve surgery but also aortic valve surgery, and it is a rare but severe complication that is treatable when diagnosed in time. Intraoperative transesophageal echocardiography is very useful in immediate diagnosis. A reconstruction of the aortic annulus with a Dacron patch is essential to treat this complication.

\section{References}

1. Martinez-Selles M, Garcia-Fernandez MA, Moreno M, Bermejo J, Dalcan JL. Echocardiographic features of left atrial dissection. Eur $J$ Echocardiogr. 2000;1:147-50.

2. Sekino Y, Sadahiro M, Tabayashi K. Successful surgical repair of left

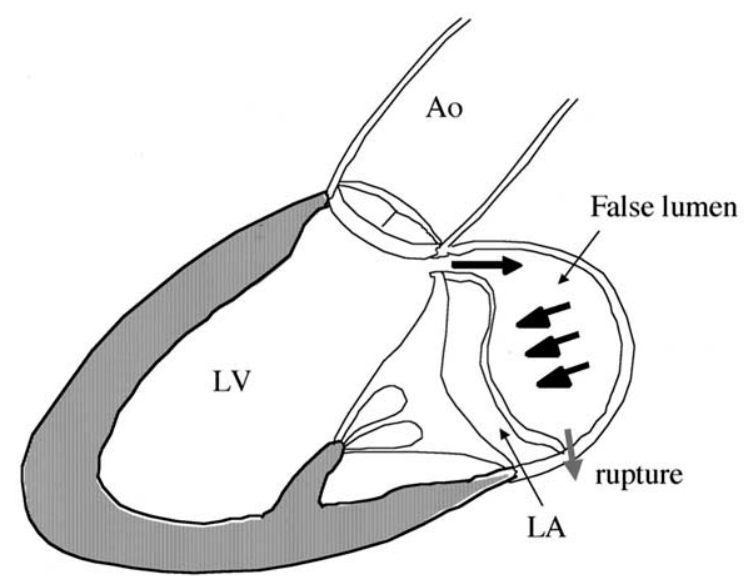

A

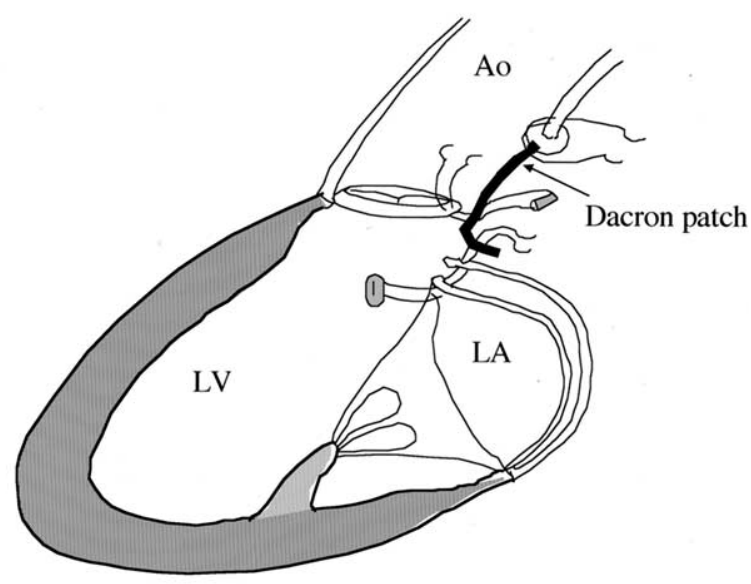

B

Figure 2. Operative findings in the patient. $A$, The entry of the left atrial dissection was caused by aortic annular disruption. $B$, The entry of the dissection and the annulus, including the aortic wall, was reconstructed with a Dacron patch, and aortic valve rereplacement was performed on the reconstructed annulus. Ao, Aorta; $L A$, left atrium; $L V$, left ventricle.

atrial dissection after mitral valve replacement. Ann Thorac Surg. 1996; 61:1528-30

3. Maeda K, Yamashita C, Shida T, Okada M, Nakamura K. Successful surgical treatment of dissecting left atrial aneurysm after mitral valve replacement. Ann Thorac Surg. 1985;39:382-4.

4. Genoni M, Jenni R, Schmid ER, Vogt PR, Turina MI. Treatment of left atrial dissection after mitral repair: internal drainage. Ann Thorac Surg. 1999;68:1394-6.

5. Ninomiya M, Taketani T, Ohtsuka T, Motomura N, Takamoto S. A rare type of left atrial dissection. J Thorac Cardiovasc Surg. 2002;124:618-20. 